\title{
La coherencia entre los marcos normativos de Naciones Unidas, la Unión Europea y el Estado español. \\ El caso de las políticas de cooperación y la dimensión exterior de la política migratoria
}

The coherence between the regulatory frameworks of United Nations, the EU and Spanish State. The case of development cooperation policies and the external dimension of migratory policy

Nerea Azkona*

Sumario: Introducción. 1. La perspectiva de las Naciones Unidas: el desarrollo humano, las migraciones internacionales y su relación con los derechos humanos. 2. El enfoque de la Unión Europea: El grado de coherencia en el cruce de las políticas de migración y cooperación al desarrollo. 3. El caso español: El nexo entre migración y desarrollo en las políticas españolas. 4. Algunas conclusiones acerca de los marcos normativos a partir del principio de Coherencia de Políticas de Desarrollo. Referencias bibliográficas.

Resumen: Este artículo tiene como objetivo clarificar la coherencia entre las políticas de cooperación y de migración del Estado español como miembro de la UE y de las Naciones Unidas. Hemos considerado el cruce de los conceptos de desarrollo, migración y cooperación al desarrollo en el ámbito de trabajo de la CPD, la cual aboga por una responsabilidad compartida en materia de migración y de desarrollo. Si el objetivo de la cooperación al desarrollo es crear condiciones que favorezcan el desarrollo de sociedades y personas en condición de pobreza y exclusión, ¿son las políticas de migración coherentes con este objetivo? ¿Qué grado de incoherencia estamos dispuestos a asumir?

Palabras clave: Coherencia, normativa, cooperación al desarrollo, política migratoria.

* Investigadora independiente. Dirección de contacto: galmena@hotmail.com 
Abstract: This article aims to clarify the coherence between the policies of cooperation and migration of the Spanish State as a member of the EU and the United Nations. We have considered the crossroads of the concepts of development, migration and cooperation development in the scope of work of the CPD, which advocates shared responsibility in the field of migration and development. If the objective of the development cooperation is the creation of conditions that favour the development of societies and people in conditions of poverty and exclusion, are the migration policies consistent with this objective? What degree of inconsistency are we willing to take?

Keywords: Coherence, regulatory frameworks, development cooperation, migratory policy. 


\section{Introducción}

Desde 2005 ha habido un cambio en la política española respecto a ciertos países africanos que, anteriormente, no estaban en sus agendas de asuntos exteriores. Los países del Mediterráneo contaban con su apoyo, con acuerdos y convenios desde hace años, pero los países de África Occidental no. Desde el Estado español, con los Planes África se comenzó a aumentar los fondos de cooperación al desarrollo, algo que la sociedad civil había estado pidiendo desde tiempo atrás. Sin embargo, lo que activó las alarmas fue la coincidencia de este hecho con el aumento de los flujos migratorios que llegaban a Europa a través del Estado español desde esos mismos países africanos.

De ahí que hayamos estudiado las normativas internacionales, europeas y españolas que han sido las herramientas de trabajo que han guiado las relaciones exteriores entre el Estado español y estos países africanos durante los últimos años, y ello con el objetivo de relacionar, bajo el principio de la Coherencia de Políticas de Desarrollo (CPD), la coherencia externa vinculando las políticas migratorias y de cooperación al desarrollo a nivel mundial (Naciones Unidas), comunitario (Unión Europea) y estatal (Estado español).

Concretamente, entre los siete objetivos que cuentan los Planes África, hemos seleccionado dos: por un lado, el que pretende luchar contra la pobreza y está ligado con la cooperación al desarrollo; y, por el otro, el que busca fomentar otro tipo de colaboración, en referencia a la cooperación en materia migratoria, para la regulación de los flujos migratorios y el combate del tráfico de personas.

A partir de todo esto, nuestra hipótesis de trabajo ha sido la existencia de cierta incoherencia entre las distintas normativas y políticas de cooperación al desarrollo comunitarias europeas y españolas en relación con las políticas de cooperación migratoria en los ámbitos en los que existen sinergias entre ellas.

Y para poder demostrarlo, hemos realizado una descripción analítica de estas políticas a partir del ámbito de trabajo de la Coherencia de Políticas a favor del Desarrollo, principio que aboga por la responsabilidad compartida de las políticas diferentes a las de cooperación al desarrollo, para que el efecto de ésta no se vea mermado por el objetivo de otras políticas más potentes.

Este estudio se ha focalizado en el caso del Estado español y de los países del África Occidental; siendo el primero uno de los principales destinos de las migraciones africanas, y los segundos los países de origen y de tránsito de las mismas. Pero como el Estado español ha cedido competencias a la Unión Europea en materia de migración a partir del Tratado de Lisboa, 
con vistas a una próxima ley migratoria común, hemos tenido que centrar el estudio, además de en las relaciones bilaterales entre estados, también en el nivel comunitario. De hecho, en el caso europeo, los Estados miembros más progresistas en materia de migración se cobijan bajo el paraguas de la Unión para justificar acciones que llevan a cabo y que no son tan progresistas como sus propias políticas (Pinyol, 2008; Carballo de la Riva, 2009).

\section{La perspectiva de las Naciones Unidas: el desarrollo humano, las migraciones internacionales y su relación con los derechos humanos}

\subsection{La motivación de las Naciones Unidas: los derechos humanos}

Dentro de las Declaraciones y Pactos que regulan los derechos humanos desde el derecho internacional, queremos detenernos en dos referencias que pertenecen a "generaciones» distintas: por un lado, los artículos 13 y 14 de la Declaración Universal de los Derechos Humanos de 1948, dentro de los derechos de primera generación; y, por el otro, la Declaración del Derecho al Desarrollo que se aprobó en 1986, dentro de la Carta Africana de Derechos Humanos y de los Pueblos, denominada Carta de Banjul y promotora de los derechos de tercera generación 1 .

El derecho a migrar viene detallado en al apartado 2 del artículo 13 de la Declaración Universal, que reza: «toda persona tiene derecho a salir de cualquier país, incluso del propio, y a regresar a su país». Derecho que podemos encontrar en el artículo 12 del Pacto Internacional de los Derechos Civiles y Políticos (en vigor desde 1976), firmado y ratificado por el Estado español en 1985.

El derecho al desarrollo también ha gozado de un cierto reconocimiento jurídico internacional para formar parte de la agenda política de las Naciones Unidas (Gómez Isa, 1998). De hecho, la Comisión de Derechos Humanos ha reconocido en distintas resoluciones el derecho al desarrollo como un derecho humano. En 1981 dicha Comisión creó un Grupo de Trabajo de Expertos Gubernamentales para que trabajase sobre la redacción de un proyecto de Declaración al respecto. Dicha Declaración, principal instrumento jurídico en materia de desarrollo, obtuvo el voto favorable

1 Es destacable que la UE asumió competencias respecto de los derechos humanos y de las libertades fundamentales y del Estado de Derecho mediante la Carta Comunitaria de Derechos de 1989; es decir, la construcción europea reposa en una concepción rigurosa de la protección de todos los derechos fundamentales. 
de 146 Estados, entre ellos el español${ }^{2}$. Aún así, sigue siendo un tema de debate el grado de normatividad jurídico-internacional que ha alcanzado como derecho humano.

Nos gustaría destacar una dimensión de este derecho, como es el deber de los Estados de llevar a cabo una cooperación para el desarrollo. En el artículo 3.3 se plasma que:

«Los Estados tienen el deber de cooperar mutuamente para lograr el desarrollo y eliminar los obstáculos al desarrollo. Los Estados deben realizar sus derechos y sus deberes de modo que promuevan un nuevo orden económico internacional basado en la igualdad soberana, la interdependencia, el interés común y la cooperación entre todos los estados, y que fomenten la observancia y el disfrute de los derechos humanos».

Cuando la Declaración hace mención a no poner obstáculos al desarroIlo, nos recuerda al principio de Coherencia de Políticas de Desarrollo, que asume que el resto de políticas no deben intervenir, incluso deben favorecer, el avance positivo de las políticas de desarrollo.

\subsection{La migración desde los derechos humanos}

La Convención Internacional sobre la Protección de los Derechos de todos los Trabajadores Migrantes y de sus Familiares, del 18 diciembre de 1990, que entró en vigor el 1 de julio de 2003, se considera el primer instrumento internacional legalmente vinculante que incorpora la serie completa de derechos humanos — derechos civiles, políticos, económicos, sociales y culturales - para todos los trabajadores migrantes y sus familiares:

«Uno de los principios más importantes establecidos en la Convención sobre los derechos de los migrantes es que todos los trabajadores y trabajadoras migrantes y sus familiares tienen derecho a que se respeten sus derechos humanos fundamentales, con independencia de su condición jurídica en el país de acogida» (Amnistía Internacional, 2006: 8-9).

El objetivo de la Convención es luchar contra la explotación y las violaciones de los derechos humanos de un grupo de población especialmente vulnerable. Así, trata de garantizar que se respeten sus derechos y que tengan igualdad de trato en sus condiciones laborales respecto a los trabaja-

2 Declaración sobre el Derecho al Desarrollo, Resolución de la Asamblea General de las Naciones Unidas, 41/128 del 4 de diciembre de 1986. 
dores estatales. En este sentido, la Convención es uno de los siete Tratados internacionales básicos de derechos humanos.

La aplicación de la Convención está condicionada a la ratificación de la legislación internacional y fundamentalmente de la estatal, lo que refleja el poder de decisión del Estado sobre la forma de garantizar los derechos de los trabajadores migrantes. El Estado español no la ha ratificado.

\subsection{El desarrollo desde los derechos humanos}

Las Naciones Unidas han promovido numerosos proyectos, planes y acuerdos para mejorar la situación de pobreza extrema (menos de un dólar al día) que arrastra a más de 1.000 millones de personas. Así lo recogen, por ejemplo, los Objetivos de Desarrollo del Milenio (ODM), resultado de la reunión que convocaba a los representantes de los Estados miembros de las Naciones Unidas en Nueva York en septiembre de 2000, denominada La Cumbre del Milenio y en la que se redactó la Declaración del Milenio.

La Comisión Europea, por su parte, ha elaborado diferentes documentos haciendo referencia a los ODM y a su consecución. En 2005 tenemos las comunicaciones de la Comisión al Consejo COM(2005) 133 final y $\operatorname{COM(2005)} 134$ final. En la segunda, la Unión Europea (UE) se compromete a promover las sinergias entre migración y desarrollo para conseguir que la inmigración sea un factor positivo para el desarrollo.

Tres años después, la Comisión Europea (2008a) elaboró una Comunicación en la que se hace referencia a la UE como socio global para el desarrollo. Trata sobre la consecución de los ODM y establece que:

«Se invita al Consejo a promover los principios de coherencia de las políticas para el desarrollo en el marco de la Agenda internacional para los ODM y la eficacia de la ayuda. Se invita al Consejo también a pronunciarse sobre las orientaciones de las políticas destinadas a aprovechar el potencial de desarrollo en materia de biocarburantes, migración e investigación» (Comisión Europea, 2008a: 12).

Por último, en 2010 y mediante la Comunicación COM(2010) 159 final, la UE propone un Plan de Acción en doce puntos en apoyo de los ODM, haciendo especial hincapié en la Coherencia de las Políticas de Desarrollo.

\subsection{De la eficacia y la calidad de la ayuda a la coherencia de políticas a favor del desarrollo}

El Consenso de Monterrey es el resultado de la Conferencia Internacional sobre la Financiación para el Desarrollo, celebrada en marzo de 2002 
en el marco de las Naciones Unidas en Monterrey (México). Ésta fue la primera Conferencia en la que fue discutida la financiación para el desarrollo.

Después de este primer acercamiento a la financiación para el desarroIlo, se siguieron otras declaraciones y eventos en los que se abordó la eficacia de la ayuda, promovidos por la OCDE, como la Declaración adoptada durante el I Foro de Alto Nivel sobre Armonización (Roma, 2003) o los principios fundamentales avanzados durante la Mesa Redonda de Marrakech sobre la gestión orientada a los resultados del desarrollo (2004), en donde se aboga por un incremento del impacto de la ayuda para reducir la pobreza y la desigualdad, acelerando el crecimiento y agilizando el cumplimiento de los ODM.

Pero si hay algún documento importante que ha guiado la actuación internacional en los últimos años es la Declaración de París sobre la Eficacia de la Ayuda al Desarrollo. Apropiación, Armonización, Alineación \& Resultados y Mutua Responsabilidad que se redactó como resultado de otro II Foro de Alto Nivel sobre la Eficacia de la Ayuda al Desarrollo, en el que los ministros de países desarrollados y en desarrollo y los directivos de instituciones multilaterales y bilaterales, reunidos el 2 de marzo de 2005, resolvieron emprender acciones de largo alcance y supervisables con vistas a reformar las maneras en las que se suministran y gestionan las ayudas. Reconocían, de este modo, que era necesario aumentar el volumen de la ayuda y de los otros recursos del desarrollo para lograr los ODM, y que era preciso incrementar al mismo tiempo de manera significativa la eficacia de la ayuda al desarrollo, así como respaldar el esfuerzo que realizan los países socios reforzando sus gobiernos y mejorando el desempeño del desarrollo.

Por último es reseñable como en el IV Foro de Alto Nivel sobre la Eficacia de la Ayuda al Desarrollo en Busan, República de Corea (2011), los líderes de países en desarrollo examinaron los progresos en la mejora del impacto y la efectividad de la ayuda al desarrollo, y los países desarrollados adquirieron nuevos compromisos para garantizar que la ayuda contribuya a reducir la pobreza y apoye el progreso hacia el cumplimiento de los ODM, con la intención de dar continuidad a la Declaración de París de 2005.

\section{El enfoque de la Unión Europea: el grado de coherencia en el cruce de las políticas de migración y cooperación al desarrollo}

\subsection{La UE: SUs motivaciones y sus políticas}

Las políticas migratorias y las de cooperación al desarrollo europeas iban en paralelo hasta que se desarrolló el enfoque global europeo de las 
migraciones en 2005, momento en el que se añade un tercer eje a las políticas migratorias que se intercepta con la cooperación al desarrollo: la dimensión exterior de las políticas migratorias.

\subsection{Las políticas migratorias de la Unión Europea: el enfoque global y su dimensión exterior}

En la UE la gestión de los flujos migratorios se ha dejado tradicionalmente en manos del particularismo de cada Estado, a su control individual, y a las legislaciones estatales de extranjería. Esta variedad normativa dificultaba el establecimiento de una política de armonización de legislaciones en el sector, y la coordinación dentro de este tema levantaba la oposición los Estados miembros ${ }^{3}$.

Un punto clave en el cambio de la mentalidad, pero lejos aún del enfoque global, fue el Acervo Schengen integrado en el marco institucional y jurídico de la UE en virtud de un Protocolo anexo al Tratado de Amsterdam sobre la desaparición de las fronteras interiores, relacionado con la migración interna ${ }^{4}$. Este hecho produjo que se volviera a abrir el debate acerca de las fronteras exteriores, ya que si se quería crear un espacio abierto al interior había que controlar aquellas.

Por eso, desde que en el Tratado de Ámsterdam (en vigor desde el año 1999) se reconociera la competencia de la UE en el ámbito de las migraciones, ésta viene esforzándose en desarrollar una política común migratoria con la finalidad de proporcionar un marco coherente para las acciones de los Estados miembros cuando actúen dentro del ámbito de sus competencias y de la propia UE (Comisión Europea, 2007b; 2008b)5. Gestionar

3 Esto se pudo apreciar por el rechazo por parte de muchos de ellos de la Decisión $85 / 381$, del 8 de julio de 1985, que fijaba el establecimiento de un procedimiento de comunicación previa y de concertación sobre las políticas migratorias, respecto de países terceros, con obligaciones de los Estados miembros de informar a la Comisión y a los restantes países sobre proyectos estatales para inmigrantes regulares o irregulares (Fernández Rozas, 2005).

4 Ya desde los años ochenta las políticas de migración habían dejado de ser un asunto exclusivamente interno de los Estados. Los Acuerdos Schengen sobre el espacio interno de libre circulación entre cinco países miembros en 1985 institucionalizaron la cooperación entre estados europeos para el control de las fronteras. La Convención Schengen se aplica desde 1995.

${ }^{5}$ En el Tratado de Ámsterdam, Título IV, Artículo 61, Visados, asilo, inmigración y otras políticas relacionadas con la libre circulación de las personas, se reconoce la responsabilidad de la UE en materia de política inmigratoria. El objetivo principal era mejorar la gestión de los flujos migratorios mediante una actuación coordinada, teniendo en cuenta la situación económica y demográfica de la UE. 
las migraciones implica ocuparse de las distintas dimensiones de este fenómeno e integrarlo en las políticas de la UE y en sus objetivos principales de prosperidad, solidaridad y seguridad (Comisión Europea, 2008b)

El debate sobre las fronteras exteriores relacionado con el espacio interior de Schengen implicó que en las conclusiones de la Presidencia del Consejo Europeo de Tampere se aconsejara incluir las migraciones dentro del Espacio de Libertad, Seguridad y Justicia (ELSJ), lo que implicaba necesariamente la realización de acciones coordinadas conjuntas por los Estados en el ámbito de la cooperación judicial, policial y en materia penal.

A su vez, se fijaron unas normas comunes para 2004 sobre tres temas: asilo, circulación de personas, e integración. Dominó el segundo punto y dentro de éste, el objetivo sobre el control de fronteras. Se desarrolló un Plan de Acción con una vigencia de cinco años que pretendía la consecución de cuatro objetivos (Martín Martínez, 2011a): desarrollar una política común de asilo y migración, crear un auténtico espacio europeo de justicia, luchar contra la delincuencia trasnacional, y lograr una acción exterior europea más fuerte.

Antes de esto, todas las iniciativas en el ámbito de la justicia así como los asuntos de interior se insertaron en el marco de la UE, pasando el tema de las migraciones del ámbito del mercado al de interior. Es decir, antes de que el enfoque global apenas tomara forma, el tema de la inmigración quedaba comprendido dentro de los asuntos referentes al mercado (mano de obra), en un primer momento; y al de interior (seguridad) en un segundo momento. Con posterioridad las migraciones procedentes de terceros Estados encontraron un marco adecuado en el nuevo ELSJ?.

En dicho Consejo se planteó por primera vez un enfoque global y por ello se ha convertido en "el embrión de la política de migraciones y desarrollo de la UE» (Carballo de la Riva, 2009: 52). Con él se consolidó la creación de un ELSJ en la UE y la necesidad de un enfoque global de las migraciones que se ocupara de los aspectos políticos, los derechos humanos y el desarrollo en terceros países y regiones. Además, se realizó un llamamiento a una mayor coherencia de las políticas internas y externas de

6 En dicho Tratado el bloque de migración y asilo deja de estar inscrito exclusivamente dentro de la cooperación intergubernamental permeando la esfera de la comunitarización de políticas. Con ello se avanza en el establecimiento de un fundamento jurídico para una política comunitaria en tal materia.

7 De hecho, en febrero de 2002, los ministros de interior decidieron crear un cuerpo europeo de guardas fronterizos, que se materializó en noviembre de 2004 con la creación de la Agencia Europea para la Gestión de las Fronteras Exteriores (FRONTEX) dedicada a la vigilancia de fronteras de la UE en cooperación con terceros países. Está operativa desde mayo de 2005. 
la UE, haciendo hincapié en la necesidad de gestionar de modo más eficaz los flujos migratorios en todas sus fases y la cooperación con terceros países para fomentar el codesarrollo.

En el Consejo Europeo de Sevilla (2002) «se planteó, en términos de política instrumental y defensiva, la política de fronteras y la adecuación coyuntural a las necesidades de trabajo» (Carballo de la Riva y Echart Muñoz, 2007: 137). Asimismo, se rescataron dos objetivos principales: por una parte, se dio prioridad absoluta al plan de lucha contra la inmigración clandestina; y, por otra parte, a la instrumentalización de la Ayuda Oficial al Desarrollo (AOD), exponiendo que:

«El Consejo Europeo recuerda que, tanto la intensificación de la cooperación económica, como el desarrollo de los intercambios comerciales, la ayuda al desarrollo y la prevención de conflictos, constituyen medios para promover la prosperidad económica de los países afectados y reducir así las causas de los movimientos migratorios. El Consejo Europeo insta a que se incluya una cláusula sobre gestión común de los flujos migratorios y sobre la readmisión obligatoria en caso de inmigración ilegal en todo futuro acuerdo de cooperación, de asociación o equivalente que la Unión Europea o la Comunidad Europea celebre con cualquier país» (Unión Europea, 2002: 10).

Quedó evidenciado que en adelante la AOD y los acuerdos de cooperación se condicionarán a la buena voluntad que habrán de demostrar los países de emigración en impedir las salidas irregulares hacia Europa y en readmitir a sus ciudadanos. Además, algunos Estados, como Reino Unido y España, reclamaron sanciones contra los países de riesgo migratorio, pero Francia y Alemania se opusieron.

Otro hito en la normativa de la UE respecto a la política migratoria se resume en el Programa de La Haya. Consolidación de la libertad, la seguridad y la justicia en la Unión Europea (2004), en el cual se precisa que:

«La política de la UE debería aspirar a ayudar a terceros países, en forma de plena asociación —utilizando si procede los fondos comunitarios existentes-, en sus esfuerzos por mejorar su capacidad de gestión de la migración y de protección de los refugiados, prevenir y combatir la inmigración ilegal, informar sobre los canales legales de migración, resolver determinadas situaciones de los refugiados proporcionando un mejor acceso a soluciones estables, crear capacidad de control de fronteras, mejorar la seguridad de los documentos y abordar el problema del retorno» (Comisión Europea, 2006a: 5).

La aplicación de este Programa abarcó el periodo comprendido entre 2005 y 2010 y tuvo como objetivo principal crear un ELSJ en torno a dos 
temas: la promoción de una política de migración común; y el desarrollo de la dimensión exterior de la política migratoria y de asilo europea.

Por fin, en diciembre de 2005 en el Consejo Europeo de Bruselas se aprobó el principio del enfoque global de las migraciones. De este modo, el Estado español se comprometió a una política de intercambio de acuerdos de readmisión a cambio de la apertura de su mercado de trabajo a cuotas de contratos para los migrantes de los países afectados.

El planteamiento de enfoque global ilustra la ambición de la UE de establecer un marco intersectorial para gestionar la migración de manera coherente a través del diálogo político y la estrecha cooperación práctica con terceros países (Comisión Europea, 2005d; 2006b; y 2007a). Los tres ejes son:

— Primer eje: perseguir la migración irregular.

- Segundo eje: promover la migración legal.

- Tercer eje: fomentar las sinergias entre la migración y el desarroIlo. Este eje complementa las estrategias sobre migración legal y migración irregular con la necesidad de diálogo y cooperación con los países de origen y tránsito de las migraciones y se corresponde con la dimensión exterior de la política migratoria.

Respecto al tercer eje, fue una declaración de intenciones la que sentó las bases de lo trabajado por parte de la Comisión desde el año 2002 a través de diversas comunicaciones, y sus respectivas líneas de financiación en torno a la sinergia entre migraciones y desarrollo, y sirvió de punto de partida para otras comunicaciones, como por ejemplo la COM(2006) 735 final, cuyo objetivo era el de analizar lo que se denomina primera fase del Planteamiento Global (acciones desarrolladas en el Mediterráneo o en África en 2006), y en la que se establecen los parámetros que responden al enfoque referido. Además, se insiste en la necesidad de elaborar una política global europea de migración basada en unos principios políticos comunes.

En junio de 2007 se reunió el Consejo Europeo de Bruselas para desarrollar una política que abarcara todos los aspectos de la migración, desde la relación entre ésta y el desarrollo hasta los aspectos internos de la UE como la migración legal, la integración, la protección de los refugiados, el control de fronteras, la readmisión, la lucha contra la migración ilegal y la trata de seres humanos. Para ello, se retoma la política global europea sobre migraciones desde el ámbito de Justicia y Asuntos de Interior, lo que nos remite a incoherencias en las comunicaciones entre las tensiones de las políticas de interior y control y la política de desarrollo.

En septiembre de 2008 el Consejo Europeo desarrolló El Pacto Europeo sobre Inmigración y Asilo (PEIA), documento político mediante el cual los Estados miembros se comprometen entre ellos en una serie de objeti- 
vos y de orientaciones estratégicas para el desarrollo de la política europea de inmigración y de asilo:

- La organización de la inmigración legal teniendo en cuenta las prioridades, las necesidades y la capacidad de acogida determinada por cada Estado miembro y el favorecimiento de la integración.

- La lucha contra la inmigración irregular, especialmente garantizando el retorno de los extranjeros en situación de irregularidad al país de origen o a un país de tránsito.

- El reforzamiento y la eficacia de los controles fronterizos.

- La construcción de la Europa del asilo.

- El establecimiento de una cooperación global con los países de origen y de tránsito favoreciendo las sinergias entre las migraciones y el desarrollo.

Entre otras medidas se prohibió a los Estados miembros las regularizaciones masivas y uno de los objetivos fundamentales del PEIA fue el de proseguir con la negociación de acuerdos de readmisión «sea a nivel comunitario, sea a título bilateral» (Unión Europea, 2008: 15).

Uno de los hechos en materia migratoria que cerró el año 2008 fue la aprobación en el Parlamento Europeo de la propuesta de una Directiva de Retorno ${ }^{8}$ que reglamenta un procedimiento común para la detención y expulsión de inmigrantes indocumentados, la que se conoce como «Directiva de la Vergüenza» y que es vinculante. Ésta es un primer paso hacia una política común de inmigración. El texto, negociado con el Consejo, promueve el retorno voluntario, introduce normas comunes para el retorno de inmigrantes ilegales procedentes de países no comunitarios, establece estándares mínimos para la retención temporal con periodos máximos de internamiento e introduce un enfoque común para la prohibición de reingreso en la UE' .

8 Directiva 2008/115/CE del Parlamento Europeo y del Consejo de 16 de diciembre de 2008 relativa a normas y procedimientos comunes en los Estados miembros para el retorno de los nacionales de terceros países en situación irregular.

9 Los Estados deben legalizar a los inmigrantes o pedirles que se vayan, para lo que se dará un periodo de salida mínimo de siete días. Si no se van, tendrán que expulsarlos, pero con garantías jurídicas y primando los intereses del menor y las familias. La retención se limita a un máximo de seis meses, que puede extenderse en ciertos casos hasta doce meses más. La Directiva define el procedimiento para la orden de expulsión (artículo 8) y las circunstancias en que pudiera darse un aplazamiento. En caso de expulsión, se plantea una prohibición de reingreso en cualquier país de la Unión Europea durante un máximo de cinco años. En ciertos casos los Estados deben incluir la prohibición de re-entrada, por ejemplo, cuando el inmigrante no se ha ido en el periodo de salida voluntaria o cuando hayan tenido que ser deportados. Además, los países de la UE tendrán que proporcionar asistencia legal a los inmigrantes sin 
Por último, cabe citar el Programa de Estocolmo. Una Europa abierta y segura que sirva y proteja al ciudadano, que fue difundido el 4 de mayo de 2010. Contempla una mayor cooperación entre los Estados miembros, siendo el control fronterizo uno de los temas más destacados. Se continúa instando a los países a adoptar una estrategia común para administrar mejor las fronteras y satisfacer las necesidades del mercado de trabajo.

El tema de la cooperación también se aborda desde la perspectiva de colaborar con terceros países a la hora de gestionar los flujos migratorios, manteniendo la política de detención y expulsión de inmigrantes que se encuentran en condición de irregularidad junto con algún incentivo para el retorno voluntario. Se sigue confiando en la Agencia FRONTEX.

\subsection{La cooperación al desarrollo de la UE: El Consenso Europeo sobre Desarrollo}

Según Martín Martínez (2011b) la política de cooperación para el desarrollo descrita en el Tratado de Lisboa se caracteriza por las llamadas tres «c»: complementariedad, ya que nos estamos refiriendo a un ámbito competencial compartido; coordinación, porque es una política trasversal que se supone que hay que tener en cuenta a la hora de aplicar otras políticas que puedan afectar a los países en desarrollo; y coherencia, entre los tres niveles de las administraciones: la política de la Unión y la de los Estados miembros deben estar en consonancia con lo acordado en Naciones Unidas.

El Tratado afirma explícitamente que la reducción y la erradicación de la pobreza son el objetivo principal de la política de la Unión en el ámbito de la cooperación para el desarrollo, y por ello la UE debe tenerlo en cuenta al aplicar las políticas que puedan afectar a los países en desarrollo (Martín Martínez, 2011b). Esto requiere que dicha política posea entidad

recursos, en función de las distintas legislaciones nacionales. Las nuevas normas obligan a tener en cuenta el mejor interés del niño. Se prevé la posibilidad de repatriar a los menores no acompañados, siempre y cuando sus familias o un centro de acogida se ocupen de ellos a su llegada. Además, los niños y las familias con menores «sólo serán internados como último recurso y ello por el menor tiempo posible» (artículo 15 bis). Los Estados miembros tendrán en cuenta la situación en el país de origen y no repatriarán a nadie cuya vida o libertad puedan estar amenazadas (artículo 5). El Parlamento Europeo decidirá con el Consejo qué países se pueden considerar "seguros» para la devolución, tomando como referencia un dictamen del Tribunal de Justicia. Más información en: http://eur-lex.europa.eu/LexUriServ/LexUriServ.do?ur $\mathrm{i}=$ OJ:L:2008:348:0098:0107:ES:PDF [Actualizado en abril de 2013]. 
propia y no constituya un mero complemento de la Política Exterior y de Seguridad Común.

La cooperación para el desarrollo y la ayuda humanitaria son competencias compartidas paralelas, es decir la UE aplica una política propia y los Estados miembros pueden hacer lo mismo, evitándose así que la actuación de la UE sea un mero complemento de las políticas estatales.

Por otra parte, el 20 de diciembre de 2005 los Presidentes de la Comisión, del Parlamento y del Consejo Europeos firmaron una Declaración de política de cooperación al desarrollo titulada Consenso Europeo sobre Desarrollo ${ }^{10}$. No tiene valor jurídico vinculante pero la amplia coincidencia alcanzada le da fuerza política.

En él se define, por primera vez en cincuenta años de cooperación, el marco de principios comunes en el que la UE y sus Estados aplicarán sus respectivas políticas de desarrollo con un espíritu de complementariedad: el principio de eficacia de la ayuda, la coordinación con los Estados miembros y los actores internacionales, y la coherencia de las políticas europeas con los objetivos del desarrollo.

Tiene el propósito de reformular las políticas de desarrollo de la UE para contribuir a erradicar la pobreza y construir un mundo más justo y pacífico. La Unión se implica de modo particular en la consecución de los ODM de las Naciones Unidas.

Podemos decir que, en la dimensión discursiva, el Índice de Desarrollo Humano (IDH) junto con los ODM son los pilares del nuevo paradigma occidental de cooperación al desarrollo. La UE se «alinea» con estos principios. Sin embargo, en la implementación de las políticas de cooperación y en sus instrumentos de financiación se olvidan estos pilares y priman los acuerdos económicos entre partes desequilibradas. De hecho, la relación entre países en cuestiones de migración y cooperación al desarrollo se ha venido concretando principalmente en acuerdos bilaterales.

10 El Consenso Europeo sobre Desarrollo intenta garantizar la coherencia entre las políticas europeas y las prioridades de desarrollo en diversos ámbitos: gobernanza; derechos humanos; Estado de Derecho; sociedad civil y democracia; desarrollo social y humano (educación y formación, igualdad de género, infancia; salud); desarrollo sostenible; medio ambiente y gestión de los recursos naturales (cambio climático, biodiversidad, agua, energía, bosques, pesca); agricultura y desarrollo rural; seguridad alimentaria; desarrollo económico y comercial; infraestructuras; etc., y también orientaciones concretas del ámbito migración y desarrollo. Más información en: http://europa.eu/legislation_summaries/development/general_ development_framework/r12544_es.htm [Actualizado en abril de 2013]. 


\subsection{El compromiso de la Unión Europea con la coherencia de políticas}

En lo que respecta al ámbito específico de la CPD, la UE ha asumido un rol significativo en este campo, dado su importante papel como donante a escala global (Comisión Europea, 2005c). Teniendo en cuenta esta responsabilidad, en 2004 el Consejo Europeo invitó a la Comisión a preparar propuestas de acción para avanzar en la CPD.

En las Conclusiones del Consejo Europeo de junio de 2004 se reiteró que la UE apoyará fuertemente los intentos de las Naciones Unidas de acelerar los avances hacia el logro de los ODM ${ }^{11}$. Con referencia a este compromiso, en las Conclusiones del Consejo de noviembre de 2004 se celebró el ofrecimiento de la Comisión a preparar propuestas específicas de actuación hasta 2015 especialmente en los ámbitos de la financiación del desarrollo y la coherencia de las políticas para el desarrollo (Unión Europea, 2004).

En el amplio contexto de las políticas de la UE, la coherencia es un compromiso multidimensional que debe ubicarse en el marco general de la estrategia para el desarrollo sostenible de la UE. Las políticas no relacionadas con el desarrollo deberían respetar los objetivos propios de estas políticas cuando ello sea posible. Las áreas prioritarias son: comercio; medio ambiente; cambio climático; seguridad; agricultura; pesca; dimensión social, empleo y trabajo digno; migraciones; investigación; sociedad de la información; transporte, y energía. Para cada una de ellas, la Comisión ha definido unas orientaciones generales o una «coherencia con los compromisos de desarrollo» (Unión Europea, 2007), que podría contribuir a acelerar el cumplimiento de los ODM, siempre que en los países en desarrollo se instauren las políticas correctas y la gobernanza adecuada y se avance de forma satisfactoria en el pleno respeto de los derechos humanos.

Asimismo, el Consenso Europeo sobre Desarrollo, adoptado en 2005, reafirma el compromiso con la CPD:

«La UE ayudará a reforzar la dimensión social de la globalización para contribuir a que todos se beneficien de sus ventajas; se esforzará por garantizar la coherencia de las políticas al servicio del desarrollo y fomentar las políticas económicas, de empleo, sociales y medioambientales, complementarias recíprocamente, en el plano mundial, regional y nacional» (Unión Europea, 2006: 9).

11 Asimismo, el Consejo Europeo confirmó en las Conclusiones del Consejo Europeo de junio de 2004 que «La Unión debe seguir reforzando su liderazgo en la lucha contra la pobreza mundial». Más información en: http://www.consilium.europa.eu/ueDocs/cms_Data/ docs/pressData/es/ec/81750.pdf [Actualizado en octubre de 2011]. 
En cuanto a las políticas de migraciones y su coherencia con las políticas de cooperación al desarrollo, en el año 2005 la UE manifestó que promovería las sinergias entre migración y desarrollo, para conseguir que la inmigración sea un factor positivo para el desarrollo. Al explorar las maneras de acelerar el avance para cumplir los ODM, la UE se comprometió a trascender las fronteras de la cooperación al desarrollo (Comisión Europea, 2005a).

En 2007 en un Informe de la Comisión sobre la CPD se ilustran los progresos ya realizados y los aún pendientes en materia de coherencia entre doce importantes políticas europeas, los mecanismos organizativos en la Unión y los objetivos de desarrollo de Europa. El informe destaca que, en general, los Estados miembros consideran que los progresos en materia de coherencia fueron más importantes en la Unión que a nivel estatal y que los conflictos de prioridades o conflictos de intereses entre los Estados miembros y entre los países en desarrollo constituyen los principales obstáculos a la CPD. A ello se añade a veces una capacidad aún insuficiente y la falta de sensibilización entre los departamentos distintos de los de desarrollo.

Por último, en 2011 se reconfiguran las prioridades de la cooperación al desarrollo europea. En el documento Programa para el cambio: Política de desarrollo de la UE se pretende incrementar el impacto de la ayuda asumiendo un enfoque más estratégico y concentrándose en menos sectores y en los países más vulnerables (Comisión Europea, 2011).

\section{El caso español: el nexo entre migración y desarrollo en las políticas españolas}

\subsection{La política migratoria española: las reformas de la Ley de Extranjería}

En el Estado español no ha existido una política migratoria hasta fechas muy recientes. De hecho, hasta 1985 no hubo una legislación específica sobre los residentes extranjeros. No obstante, la Constitución Española de 1978 incluye un artículo sobre los derechos de los extranjeros (artículo 13), así como un principio de competencias exclusivas del Estado en la gestión de la inmigración. De este modo, de acuerdo con el artículo 149, todas las cuestiones relacionadas con la inmigración, el asilo, la nacionalidad, los pasaportes, las fronteras y los extranjeros quedan bajo la responsabilidad de las instituciones centrales del Estado.

La transformación del fenómeno migratorio que comienza a producirse en el Estado español a finales de los ochenta y en los años posterio- 
res, mostró la necesidad de una Ley que se adaptara a las nuevas circunstancias de un país de inmigración (Mullor, 2011).

La primera generación de leyes de inmigración incluía la Ley Orgánica 7/1985, de 1 de julio, sobre Derechos y Libertades de los Extranjeros en España de 198512, llamada Ley de Extranjería; y la Ley 5/1984, de 26 de marzo, Reguladora del Derecho de Asilo y la Condición de Refugiado, denominada Ley de Asilo que se aprobó en $1984^{13}$ y se desarrolló legislativamente al año siguiente.

Este primer conjunto de legislaciones en la materia representó las líneas inspiradoras que sustancialmente han seguido vigentes, haciendo un especial énfasis en el control de los flujos migratorios y en la regulación de los requisitos exigidos para la presencia de extranjeros en el territorio estatal.

En enero de 2000 se realizó una reforma de la Ley de Extranjería de 1985, que la derogó íntegramente. Esta Ley es la que está en vigor en la actualidad pero ha sufrido tres importantes modificaciones (en el mismo año en la que fue creada, 2000; en el 2003; y en el 2009).

Así, se aprobó la nueva Ley Orgánica 4/2000, de 11 de enero, sobre Derechos y Libertades de los Extranjeros en España y su Integración Social que se convirtió en la norma que regula la entrada de los extranjeros provenientes de terceros países en el territorio español, así como los derechos y las libertades que se les reconocen ${ }^{14}$. Esta reforma mostraba una clara vocación integradora que no contenía la regulación anterior.

Sin embargo, el cambio de Gobierno del PSOE al PP, a través de las Elecciones Generales del 2000, condujo a una primera reforma restrictiva de la Ley de Extranjería15. De este modo, a partir de la Ley Orgánica 8/2000, el estatus legal de la irregularidad se endureció; el régimen

12 Ley Orgánica 7/1985, de 7 de julio, sobre Derechos y Libertades de los Extranjeros en España (vigente hasta el 1 de febrero de 2000). Para ver más en: http://extranjeros. empleo.gob.es/es/normativajurisprudencia/Nacional/RegimenExtranjeria/RegimenGeneral/ documentos/antecedentesNormativos/ley7_1985.pdf [Actualizado en mayo de 2013].

13 Ley 5/1984, de 26 de marzo, Reguladora del Derecho de Asilo y la Condición de Refugiado, en su redacción dada por la Ley 9/1994, de 19 de mayo (versión consolidada). Para ver más en: http://extranjeros.empleo.gob.es/es/normativajurisprudencia/Nacional/RegimenAsilo/ documentos/Refundido_Asilo_94.pdf [Actualizado en mayo de 2013].

14 Ley Orgánica 4/2000, de 11 de enero, sobre Derechos y Libertades de los Extranjeros en España y su Integración Social. Para ver más: http://noticias.juridicas.com/base_datos/ Admin/lo4-2000.html [Actualizado en mayo de 2013].

15 Ley Orgánica 8/2000, de 22 de diciembre, de reforma de la Ley Orgánica 4/2000, de 11 de enero, sobre Derechos y Libertades de los Extranjeros en España y su Integración Social. Para ver más: http://extranjeros.empleo.gob.es/es/NormativaJurisprudencia/Nacional/ RegimenExtranjeria/RegimenGeneral/documentos/Ley_Organica_8-2000.pdf [Actualizado en mayo de 2013]. 
de sanciones se hizo más severo; y el poder otorgado al Ejecutivo para desarrollar el contenido de la Ley se incrementó de forma importante. Se justificó esta reforma en el preámbulo de la Ley diciendo que la anterior no se ajustaba a los compromisos internacionales suscritos por España en el marco de la Unión Europea, y que la anterior Ley sólo contemplaba la situación entonces actual sin tener en cuenta los años venideros.

En 2003, a causa de la vuelta del PSOE al Gobierno Central, la Ley de Extranjería fue reformada de nuevo mediante dos leyes: la Ley Orgánica 11/2003 que hacía referencia a una serie de medidas concretas en materia de seguridad ciudadana, violencia doméstica e integración social de los extranjeros que modificó los artículos 57, 61 y 62 de la L.O. 4/200016; y la Ley Orgánica 14/2003, que se encuentra en relación directa con la Ley Orgánica 4/200017.

La última modificación de la Ley de Extranjería se debe a la Ley Orgánica 2/2009, de 11 de diciembre, sobre Derechos y Libertades de los Extranjeros en España y su Integración Social, la cual incorporó las Directivas Europeas sobre inmigración al derecho interno y se adaptó a las competencias de acogida e integración previstas en los Estatutos de Autonomía ${ }^{18}$.

Dentro de este contexto de cambios constantes del marco normativo se ha desarrollado la política de migración española.

\subsection{La política de cooperación al desarrollo española: la Ley de Cooperación Internacional para el Desarrollo y la Coherencia de Políticas de Desarrollo}

En 1991 el Estado español se incorporó al Comité de Ayuda al Desarrollo (CAD) de la OCDE, hecho que le consolidó como país donante dentro del sistema internacional de cooperación. Sin embargo, durante estos años también empezaron a manifestarse los primeros síntomas de un

16 Ley Orgánica 11/2003, de 29 de septiembre, de Medidas Concretas en Materia de Seguridad Ciudadana, Violencia Doméstica e Integración Social de los Extranjeros. Para ver más en: http://boe.vlex.es/vid/concretas-ciudadana-violencia-domestica-16035861 [Actualizado en mayo de 2013].

17 Ley Orgánica 14/2003, de 20 de noviembre, de Reforma de la Ley orgánica 4/2000, de 11 de enero, sobre Derechos y Libertades de los Extranjeros en España y su Integración Social. Para ver más en: http://www.boe.es/boe/dias/2003/11/21/pdfs/A41193-41204.pdf [Actualizado en mayo de 2013].

18 Ley Orgánica 2/2009, de 11 de diciembre, de reforma de la Ley Orgánica 4/2000, de 11 de enero, sobre Derechos y Libertades de los Extranjeros en España y su Integración Social. Para ver más en: http://www.boe.es/boe/dias/2009/12/12/pdfs/BOE-A-2009-19949.pdf [Actualizado en mayo de 2013]. 
modelo en crisis, relacionado con la rápida transición de país receptor a país donante, además del contexto de crisis que ya estaba viviendo dicho sistema desde los principios de los años noventa (Boza y Báez, 2004).

En 1985 fue aprobada una nueva estructura orgánica del Ministerio de Asuntos Exteriores, que supuso la creación de la Secretaría de Estado para la Cooperación Internacional y para Iberoamérica (SECIPI). El Real Decreto 1527/1988, de 11 de noviembre, reestructuró la SECIPI, y creó la Agencia Española de Cooperación Internacional (AECI), a la cual se le encomienda propiciar el desarrollo económico y el progreso social, cultural, institucional y político de los Países en Vías de Desarrollo y, en especial, de los de Iberoamérica, así como favorecer el estrechamiento de los lazos de entendimiento y cooperación entre los países desarrollados, especialmente de los integrados en las Comunidades Europeas ${ }^{19}$.

En julio de 1998 se adoptó la Ley de Cooperación Internacional para el Desarrollo (LCID), lo que implicó un salto significativo en la política de cooperación que había ido adquiriendo un peso cada vez mayor en el conjunto de la política exterior española. A diferencia de la Ley de Extranjería, esta otra no ha sufrido cambios a lo largo de tiempo y tiene competencias cedidas a las Comunidades Autónomas.

En 1996, se reestructuró la Agencia y en 2008 pasó a denominarse de forma oficial Agencia Española de Cooperación Internacional para el Desarrollo (AECID).

El ámbito de trabajo de la Coherencia de Políticas de Desarrollo (CPD) cada vez está más visible en los documentos españoles relacionados con la cooperación; aunque «a pesar de la atención dedicada por algunos países donantes a los aspectos relacionados con la coherencia de políticas, en España apenas si se ha iniciado la reflexión sobre esta materia» (Alonso, 2003: 235) . $^{20}$.

En 2007 el CAD hizo un examen a España en el que resaltaron las principales recomendaciones en materia de cooperación. Refiriéndose a la

19 Real Decreto 1527/1988, de 11 de noviembre, por el que se reestructura la Secretaría de Estado para la Cooperación Internacional y para Iberoamérica, con refundición de los Organismos autónomos adscritos a la misma. Para ver más: https://www.boe.es/diario_boe/txt. php?id=BOE-A-1988-29179 [Actualizado en mayo de 2013].

20 En 2004 se aprobó un Real Decreto por el cual se dotaba al Consejo de Cooperación al Desarrollo del mandato de «informar anualmente sobre el cumplimiento del principio de coherencia en la actuaciones de cooperación realizadas por los diversos organismos de la Administración General del Estado. Este informe será remitido a la comisión de Cooperación Internacional para el Desarrollo del Congreso de los Diputados». Real Decreto 2217/2004, de 26 de noviembre, sobre competencias, funciones, composición y organización del Consejo de Cooperación al Desarrollo. Para ver más en: http://www.boe.es/buscar/act.php?id=BOEA-2004-20119 [Actualizado en mayo de 2013]. 
CPD alabó el apoyo político que se estaba dando a este ámbito, pero enfatizó la necesidad de un uso más sistemático y estratégico que garantice su avance (CAD, 2007).

\subsection{La consolidación de la política de cooperación española y su relación con la migración: el III Plan Director de la Cooperación Española}

El III Plan Director de la Cooperación Española (2009/2012) (IIIPD) incorpora, por primera vez, un sector específico dedicado exclusivamente a las migraciones y el desarrollo. Parte de la base de que la lucha contra la pobreza es un fin en sí mismo y no tiene relación de causalidad inmediata con las migraciones; y del convencimiento de que la migración debe ser fruto de una libre decisión, de una opción personal y no de la necesidad.

Desde estas premisas el IIIPD tiene en este sector como objetivo general la promoción de los efectos positivos entre migración y desarrollo, a través de cinco fines específicos:

- Apoyar a los países de origen y tránsito en el establecimiento de marcos y mecanismos públicos para la gestión y la información de las migraciones, que maximicen los efectos positivos y minimicen las consecuencias negativas de la migración sobre el desarrollo, asegurando el respeto y la promoción de los derechos humanos.

- Promover espacios para el diálogo, la coordinación y articulación de la acción conjunta sobre migraciones y desarrollo entre sector público, sector privado, asociaciones de migrantes y organizaciones de la sociedad civil tanto, en los países de origen como en el de destino, con especial atención a la creación de alianzas transnacionales e iniciativas endógenas para la puesta en marcha de iniciativas de codesarrollo.

- Fomentar en los países de origen la formación destinada a la creación y mejora de las capacidades profesionales para el fomento de capacidades y oportunidades, orientado a la inserción efectiva en el mercado laboral.

- Impulsar la coherencia y las contribuciones positivas que las políticas públicas del Estado español pueden tener sobre los objetivos de desarrollo en los países de origen y tránsito de la migración.

- Generar y gestionar conocimiento y mejorar la capacidad disponible en el capital humano de la cooperación española para identificar, analizar e impulsar ámbitos de actuación e iniciativas concretas 
en el marco de migración y desarrollo, así como apoyar el conocimiento generado en los países de origen y las asociaciones de inmigrantes presentes en el territorio español.

A través de estos cinco objetivos estratégicos en los que está distribuido este sector, el IIIPD busca la coherencia de todas las actuaciones bajo el estricto respeto de los derechos humanos de las personas migrantes en todas las fases del proceso, tanto en los países de origen, tránsito y destino, haciendo especial énfasis en el ámbito multilateral y en la protección de las personas especialmente vulnerables, como mujeres y niños, y en cumplimiento de los consensos europeos e internacionales de los que España forma parte.

Este discurso es coherente con la política de desarrollo de la UE, caracterizada por el Consenso del Desarrollo Europeo; y con la Declaración del Milenio de las NN.UU.

Sin embargo, la AOD puede convertirse en la moneda de cambio para control de los flujos migratorios, a causa de la debilidad de la política de cooperación con respecto a la de migración.

Es por esto que más que incluir los intereses de las políticas migratorias dentro de las estrategias de cooperación al desarrollo (lo que viene siendo la dimensión exterior de la política migratoria) deberían tenerse en cuenta también los retos de la cooperación al desarrollo en la política migratoria. Es decir, partir de la cooperación al desarrollo, poniéndola en un lugar privilegiado, y no centrarse en la política migratoria que tiene objetivos con más repercusión que la cooperación.

Esta idea se encuentra en todos los discursos de los distintos agentes de cooperación que hemos visto hasta el momento. No obstante, las acciones no acompañan a las palabras y a los compromisos políticos.

Las normativas de la UE y del Estado español en materia de desarrollo están alineadas con las tesis de las NN.UU. aunque su implementación se desvía del objetivo del desarrollo. Esta diferencia se puede observar en el lenguaje de la Comisión Europea; cuando las comunicaciones son relativas a las actividades de las NN.UU. o los ODM se habla de "gestión de flujos», mientras que cuando se refieren a comunicaciones relativas a los Estados miembros hablan de «control de flujos».

\subsection{La confluencia entre la cooperación en materia migratoria y la cooperación al desarrollo española con África}

La incorporación de instrumentos de política exterior a la política de inmigración introduce un cambio que no entraña una alteración signifi- 
cativa en el diseño de la política de inmigración ni se asocia con la modificación de su marco legal, ya que aquellos no se activan necesariamente a partir de la promulgación de leyes internas, sino a través de la adopción de acuerdos de carácter internacional o supraestatal.

El reparto de competencias en materia migratoria dentro del Estado español, a partir de estos nuevos instrumentos, abarca, sobre todo, tres Ministerios que se corresponden con los tres pilares del enfoque global de la política migratoria de la UE21:

- El Ministerio del Interior, que asume las responsabilidades relativas a seguridad, control de fronteras y lucha contra la inmigración irregular (a veces acompañado del Ministerio de Defensa).

- El Ministerio de Trabajo o de Empleo, que tiene encomendadas las competencias de la migración legal, en concreto en el ámbito de la contratación en origen y la integración de los inmigrantes en el Estado.

- El Ministerio de Asuntos Exteriores y Cooperación (MAEC), con las competencias para promover las relaciones y el diálogo con terceros países, así como la cooperación al desarrollo de países empobrecidos.

Como podemos observar, el diseño de la actual política española de inmigración es una materialización del enfoque global en materia de gestión de las migraciones promovido por la Unión Europea desde 2005, que se sustenta en una amalgama de asuntos de interior, cooperación al desarrollo y relaciones exteriores. De este modo, el Estado español responde a un fenómeno generador de interdependencias con un enfoque en sintonía con los discursos acerca de la gobernanza (Delgado, 2010).

La externalización de la frontera sur por parte del Estado español vino, por una parte, de la mano de los Planes África ${ }^{22}$ y su objetivo de fomentar la cooperación para regular los flujos migratorios procedentes de África Subsahariana; y, por la otra, de la firma de los acuerdos de nueva generación. Así, el Gobierno español optó por reforzar las relaciones bilaterales con los países de origen y tránsito migratorio africano, iniciando distintas rondas negociadoras, con el objetivo de enlazar las políticas de inmigración con las de desarrollo, incorporando elementos de cooperación técnica con terceros países (Pinyol, 2008).

21 Debemos tener en cuenta que en los periodos 2006/2008 y 2009/2012 ha habido dos Gobiernos en España de distinta orientación, lo que conlleva un cambio en el nombre de los Ministerios y traslados de funciones de unos a otros.

22 I Plan África (IPA) ejecutado de 2006 a 2008 y el II Plan África (IIPA) vigente desde 2009 hasta 2012. 
Los Planes África han articulado gran parte de la política española de migración y desarrollo y del despliegue de la acción exterior en el continente a partir de: las misiones diplomáticas; la apertura de nuevas oficinas de cooperación; el aumento de la AOD; y las firmas de acuerdos que corresponden a la integralidad con la que se tratan los flujos migratorios (Carballo de la Riva, 2009). Aparecen como planes de la política exterior española centrados en temas de cooperación internacional y aparentemente guiados por consideraciones al margen de la política de inmigración. No obstante, a pesar de ser herramientas de exteriores podemos decir que son, también, instrumentos de la política de control de flujos migratorios.

El objetivo compartido entre la cooperación en materia migratoria y la cooperación al desarrollo es muy fácil de determinar. Los ODM, gracias a la $C P D$, tienen que estar en el punto de mira de todas las políticas si quieren ser conseguidos para 2015. De hecho, el IPA considera que «el pilar fundamental de este compromiso [de los ODM] con África Subsahariana es la cooperación para el desarrollo» (MAEC, 2006: 11).

Con todo, uno de los objetivos claves de dichas políticas se refiere al esfuerzo que hay que efectuar para luchar contra la inmigración irregular mediante el incremento de la cooperación internacional y la acción concertada, idea que procede del enfoque global europeo y que consigue que las políticas de cooperación sean condicionadas por políticas que son más fuertes, como es el caso de las migratorias.

Esto quiere decir que las acciones que conllevan las herramientas españolas del MAEC no respetan el objetivo de la cooperación al desarrollo a pesar de ser específicas de cooperación. Tampoco consideran los ODM, aunque el primer objetivo que se establece en el IPA es «la lucha contra la pobreza y el cumplimiento de los ODM», pero gracias a la «transparencia obscena» que envuelve a dichos Planes, ya ni siquiera esconden los intereses españoles en África, lo que hace que la consecución de ese primer objetivo no sea factible (Romero, 2006).

Encontramos incoherencias entre distintos aspectos de la misma política pública, como es la cooperación al desarrollo y la cooperación en materia migratoria. Este nexo se establece en dos objetivos de cada uno de los Planes África. El que se refiere al fomento de la lucha contra la pobreza y el logro de los ODM se corresponde con el Objetivo 2 de ambos Planes. En cambio, la cooperación en materia migratoria queda patente en el Objetivo 3 del IPA, que pretende "fomentar la cooperación para ordenar los flujos migratorios»; y en el Objetivo 4 del IIPA, que aboga por la «consolidación de la asociación con África en materia migratoria».

En este sentido, el IPA propone soluciones a la migración irregular africana haciendo referencia al enfoque global de la política migratoria, pero 
en el texto olvidan hacer mención al tercer pilar con el que se pretende fomentar las sinergias entre la migración y el desarrollo. De este modo se aspira a:

«Adoptar en materia migratoria (...) un enfoque global que tenga presentes las causas de la inmigración, los efectos positivos que una inmigración regular y ordenada pueden tener tanto para los países de origen como para España como país de destino, y las circunstancias en las que se produce la inmigración irregular, con efectos devastadores para países de origen, tránsito y destino de los flujos migratorios» (MAEC, 2006: 34-35).

Ésta es la estrategia de la UE en materia migratoria dentro de un instrumento de acción exterior español. Es decir, que las ideas de «a más desarrollo, menos migración» y la de "colaborar con los que nos ayudan», se encuentran en instrumentos relacionados con las políticas migratorias de la UE y en herramientas del MAEC del Estado español.

Esto choca de lleno con las ideas que subyacen en informes y actividades de las NN.UU., como el hecho de considerar a las migraciones como un factor de desarrollo; o la obligatoriedad que tienen los Estados de cooperar para que se cumpla el derecho al desarrollo de otros países.

\section{Algunas conclusiones acerca de los marcos normativos a partir del principio de coherencia de políticas de desarrollo}

Los Tratados, Declaraciones y Convenciones de las Naciones Unidas hacen referencia a los derechos humanos. Por un lado, no encontramos con el derecho a migrar; y, por el otro, con el derecho a no migrar, que es la otra cara del derecho al desarrollo. El Estado español ha ratificado los Pactos de las NN.UU. respecto a los derechos de primera y segunda generación, así como el derecho al desarrollo, y por ello debe tener en cuenta su aplicación a la hora de implementar sus políticas.

Recordamos que los Estados que han ratificado el derecho al desarrollo tienen el deber de cooperar con el resto de países hasta lograrlo. Esto, junto con el Objetivo 8 de los ODM, choca frontalmente con el espíritu que engloba a los Planes África del Estado español, ya que la idea que subyace a estas políticas: "colaborar con quien nos ayuda», van en contra de la misión de NN.UU.

El papel de la UE ante las NN.UU es diferente que el que asume frente a sus Estados miembros. Encontramos que hay dos líneas discursivas:

- La que está enfocada a las directrices internacionales y que se encuentra alineada con los ODM. 
- La dirigida a su propia política comunitaria de migración, que dista de la otra visión y en la que prima el control de fronteras (no a la gestión) y en la que el dialogo político al que se evoca no es para mejorar la política de visados sino que para implementar la política de repatriación.

Además no es un diálogo que sea igualitario ya que en las políticas no se tienen en cuenta las relaciones de poder que existen entre países y entre bloques de países.

Es decir, la UE es incoherente con el discurso internacional en la implementación de sus políticas, pero no lo es a nivel discursivo si tenemos en cuenta las comunicaciones de la Comisión Europea, manteniéndose más coherente en las comunicaciones que hacen referencia al propio organismo internacional.

Esto es, dentro de las políticas de desarrollo en las dimensiones que hacen referencia a los discursos encontramos que las NN.UU. (Declaración del Milenio, ODM), la UE (Consenso Europeo del Desarrollo) y el Estado español (Planes Directores de la Cooperación Española) mantienen una línea coherente en sus documentos.

Sin embargo, el caso del Estado español es más peligroso, ya que la política exterior está alineada e inspirada por el enfoque global de la política migratoria europea y su dimensión exterior. En este sentido, la política exterior española es coherente con la dimensión exterior de la política migratoria europea, en cada uno de los ejes del enfoque global, pero existen tensiones entre los tres niveles así como entre las políticas migratorias y los intereses de desarrollo de los países de origen. Esto se convierte en un tema preocupante, cuando se constata el riesgo de instrumentalizar la AOD. Por lo tanto, la perspectiva integral de las migraciones está más presente en el discurso político que en las políticas.

Respecto al Estado español, la política de interior y de exterior está empeñada en el control de los flujos migratorios que provienen de África, y los instrumentos que se crean giran en torno a esta preocupación. Dentro de la política de interior puede que no acarree demasiadas incoherencias, pero ambos planes expuestos del MAEC evidencian esta prioridad sobre el interés solidario por el desarrollo del continente africano.

En este contexto de incoherencia de políticas y de asociacionismo interesado, resulta difícil la promoción de dicho desarrollo, si lo que se persigue es intensificar las relaciones económicas y políticas internacionalizando las empresas españolas y externalizando la frontera sur de Europa. 


\section{Referencias bibliográficas}

Alonso, J.A. (2003). Coherencia de políticas y ayuda al desarrollo: el caso español, en J.A. Alonso y V. Fitzgerald (eds.). Financiación del desarrollo y coherencia en las políticas de los donantes. Madrid: Catarata e Instituto Complutense de Estudios Internacionales (ICEI), pp. 235-266.

Azkona, N. (2013). La coherencia de los Planes África a examen: La relación entre los flujos de migración y los fondos de cooperación al desarrollo entre África Occidental y la Unión Europea. Leioa (Bizkaia), Tesis Doctoral.

Azkona, N. y Sagastagoitia, J. (2011). Políticas de control migratorio y de cooperación al desarrollo entre España y África Occidental durante la ejecución del primer Plan África, Bilbao: Fundación Alboan y Entreculturas. http://www.entreculturas.org/files/documentos/estudios_e_informes/ InformeControlMigratorioyAOD_2011.pdf?download

Amnistía Internacional (2006). Vivir en las sombras: una introducción a los derechos humanos de las personas migrantes, Madrid: Editorial Amnistía Internacional (EDAI). http://web.amnesty.org/library/Index/ESLPOL330062006

Boza, J. y Báez, J.M. (2004). La cooperación al desarrollo en España. El modelo español de Ayuda Oficial al Desarrollo. XVIII Reunión Anual de la Asociación Internacional de Economía Aplicada (ASEPELT), Universidad de León. http://www.asepelt.org/ficheros/File/Anales/2004\%20-\%20Leon/ comunicaciones/Boza\%20y\%20Baez.doc

CAD (2007). Spain. Development Assistance Committe (DAC). Paper Review. París, OCDE. http://www.oecd.org/development/peer-reviews/SpainPR.pdf

Carballo de la Riva, M. (2009). Migraciones y desarrollo: la incidencia de Africa subsahariana en la agenda política. Documento de Trabajo n. ${ }^{\circ} 13$. Madrid: Instituto Universitario de Desarrollo y Cooperación y Universidad Complutense. http://pendientedemigracion.ucm.es/info/IUDC/img/articulos/ publicaciones/MIGRACIONES_Y_DESARROLLO_DT13.pdf

Carballo de la Riva, M. y Echart, E. (2007). Migraciones y desarrollo. Estrategias de acción en el Sahel Occidental. Madrid: Fundación Internacional para Iberoamérica de Administración y Políticas Públicas e Instituto Universitario de Desarrollo y Cooperación. http://pendientedemigracion.ucm.es/info/IUDC/ img/biblioteca/FIIAPP_Migraciones_sahel.pdf

Comisión Europea (2005a). Comunicación de la Comisión al Consejo y al Parlamento Europeo Acelerar el avance para cumplir los Objetivos de DesarroIlo del Milenio-Financiación para el Desarrollo y Eficacia de la Ayuda, Bruselas, 7-10-2005 (COM(2005) 133 final). http://eur-lex.europa.eu/LexUriServ/ LexUriServ.do?uri=COM:2005:0133:FIN:ES:PDF

Comisión Europea (2005b). Comunicación de la Comisión al Consejo, al Parlamento Europeo y al Comité Económico y Social Europeo Coherencia de las Políticas en favor del Desarrollo-Acelerar el avance para cumplir los Objetivos de Desarrollo del Milenio. Bruselas, 12-4-2005 (COM(2005) 134 final). http://eur-lex.europa.eu/LexUriServ/LexUriServ. do?uri=COM:2005:0134:FIN:ES:PDF 
Comisión Europea (2005c). Comunicación de la Comisión al Consejo, al Parlamento Europeo y al Comité Económico y Social Europeo Estrategia de la UE para África: hacia un pacto euroafricano para acelerar el desarrollo en África. Bruselas, 12-10-2005 (COM(2005) 489 final). http://ec.europa. eu/development/icenter/repository/COMM_PDF_COM_2005_0489_F_ES_ ACTE.pdf

Comisión Europea (2005d). Comunicación de la Comisión al Consejo y al Parlamento Europeo Prioridades de actuación frente a los retos de la inmigración: Primera etapa del proceso de seguimiento de Hampton Court. Bruselas, 30-11-2005 (COM(2005) 621 final). http://eur-lex.europa.eu/ LexUriServ/site/es/com/2005/com2005_0621es01.pdf

Comisión Europea (2006a). Comunicación de la Comisión al Parlamento Europeo y al Consejo Programa temático de cooperación con terceros países en materia de migración y asilo. Bruselas, 25-1-2006 (COM(2006) 26 final). http://eur-lex.europa.eu/LexUriServ/LexUriServ. do? uri=COM:2006:0026:FIN:ES:PDF

Comisión Europea (2006b). Comunicación de la Comisión al Consejo y al Parlamento Europeo El Planteamiento global sobre la migración un año después: Hacia una política global europea en materia de migración. Bruselas, 30 11-2006 (COM(2006) 735 final). http://ec.europa.eu/development/icenter/ repository/COMM_PDF_COM_2006_0735_F_ES_ACTE.pdf

Comisión Europea (2007a). Comunicación de la Comisión al Parlamento Europeo, al Consejo, al Comité Económico y Social Europeo y al Comité de las Regiones. Aplicación del «Planteamiento global sobre la migración»a las regiones orientales y sudorientales vecinas de la Unión Europea. Bruselas, 16-5-2007 (COM(2007) 247 final). http://eur-lex.europa.eu/LexUriServ/ LexUriServ.do? uri=COM:2007:0247:FIN:ES:PDF

Comisión Europea (2007b). Comunicación de la Comisión al Parlamento Europeo, al Consejo, al Comité Económico y Social Europeo y al Comité de las Regiones Una política común de inmigración. Bruselas, 5-12-2007 (COM(2007) 780 final). http://eur-lex.europa.eu/LexUriServ/LexUriServ. do? uri=COM:2007:0780:FIN:ES:PDF

Comisión Europea (2008a). Comunicación de la Comisión al Parlamento Europeo, al Consejo, al Comité Económico y Social Europeo y al Comité de las Regiones La UE como socio global para el desarrollo. Acelerar los avances hacia los Objetivos de Desarrollo del Milenio. Bruselas, 9-4-2008 (COM(2008) 177 final). http://eur-lex.europa.eu/LexUriServ/LexUriServ. do? uri=COM:2008:0177:FIN:ES:PDF

Comisión Europea (2008b). Comunicación de la Comisión al Consejo, al Parlamento Europeo, al Comité Económico y Social Europeo y al Comité de las Regiones Una política común de emigración para Europa: principios, medidas e instrumentos. Bruselas, 17-6-2008 (COM(2008) 359 final). http://eur-lex.europa.eu/LexUriServ/LexUriServ. do? uri=COM:2008:0359:FIN:ES:PDF

Comisión Europea (2010). Comunicación de la Comisión al Parlamento Europeo, al Consejo, al Comité Económico y Social Europeo y al Comité de las 
Regiones Plan de acción de la UE en doce puntos en apoyo de los Objetivos de Desarrollo del Milenio. Bruselas, 21-4-2010 (COM(2010) 159 final). http://www.pazysolidaridad.ccoo.es/ficheros/documentos/59_08\%20 Plan \% 20de \% 20Acci\%F3n\%20de \%20la \% 20UE \% 20en \% 2012\% 20 puntos\%20en\%20apoyo\%20a\%20los\%200DM.pdf

Comisión Europea (2011). Comunicación de la Comisión al Parlamento Europeo, al Consejo, al Comité Económico y Social Europeo y al Comité de las Regiones Incremento del impacto de la política de desarrollo de la UE: Programa para el cambio. Bruselas, 13-10-2011 (COM(2011) 637 final). http:// ec.europa.eu/europeaid/what/development-policies/documents/agenda_ for_change_es.pdf

Delgado, L.M. (2010). La acción exterior como innovación en la política española de inmigración. CIDOB d'Afers Internacionals, n. ${ }^{\circ}$ 91, pp. 6385. http://www.cidob.org/es/content/download/24685/304783/file/3_ LETICIA+DELGADO.pdf

Fernández Rozas, J.C. (2005). Génesis y constitucionalización de la política migratoria en la Unión Europea. Anuario Español de Derecho Internacional Privado (AEDIPr), Tomo V, pp. 223-251.

Gómez Isa, F. (1998). El derecho al desarrollo: entre la justicia y la solidaridad. Serie Derechos Humanos, n. ${ }^{\circ}$ 1. Bilbao: Universidad de Deusto.

MAEC (2006). Plan África 2006-2008. Madrid: Ministerio de Asuntos Exteriores y Cooperación. http://www.maec.es/SiteCollectionDocuments/Home/ LIBROPLANAFRICA.pdf

Martín Martínez, M.M. (2011a). El espacio de libertad, seguridad y justicia, en J. Alcaide y R. Casado (eds.). Curso de Derecho de la Unión Europea. Madrid: Tecnos, pp. 369-387.

Martín Martínez, M.M. (2011b). La acción exterior de la Unión Europea, en J. Alcaide y R. Casado (eds.). Curso de Derecho de la Unión Europea. Madrid: Tecnos, pp. 389-416.

Mullor, M. (2011). Inmigrantes subsaharianos. Una aproximación a las claves de la exclusión. Madrid: Asociación Círculo Africano y ONGD África Activa. http://www.africafundacion.org/IMG/pdf/Mullor_-_Inmigrantes_ subsaharianoa.pdf

Pinyol, G. (2008). La política de inmigración española como un nuevo instrumento de acción exterior, en E. Barbé (coord.). España en Europa 20042008, Monografías del Observatorio de Política Exterior Europea, n. ${ }^{\circ} 4$, Barcelona: Institut Universitari d'Estudis Europeus. http://www.iuee.eu/pdfpublicacio/127/8EvpC9JCPnW7kQrlO571.PDF

Romero, E. (2006). Quién invade a quién. El Plan África y la inmigración. Oviedo: Cambalache.

Unión Europea (2002). Consejo Europeo de Sevilla. http://www.consilium. europa.eu/uedocs/cms_data/docs/pressdata/es/ec/72639.pdf

Unión Europea (2004). Conclusiones de la Presidencia del Consejo Europeo de junio de 2004. http://www.consilium.europa.eu/ueDocs/cms_Data/docs/ pressData/es/ec/81750.pdf 
Unión Europea (2006). Consejo Europeo de Bruselas. 15914/1/05, REV 1. http://register.consilium.europa.eu/pdf/es/05/st15/st15914-re01.es05.pdf Unión Europea (2007). Consejo Europeo de Bruselas. 16616/1/07 REV 1. http:// www.consilium.europa.eu/ueDocs/cms_Data/docs/pressData/es/ec/97678. pdf

Unión Europea (2008). Pacto europeo sobre migración y asilo. http://register. consilium.europa.eu/pdf/es/08/st13/st13440.es08.pdf 


\section{Derechos de autor (Copyright)}

Los derechos de autor de esta publicación pertenecen a la editorial Universidad de Deusto. El acceso al contenido digital de cualquier número del Anuario de Acción Humanitaria y Derechos Humanos (en adelante Anuario) es gratuito inmediatamente después de su publicación. Los trabajos podrán descargarse, copiar y difundir, sin fines comerciales y según lo previsto por la ley. Así mismo, los trabajos editados en el Anuario pueden ser publicados con posterioridad en otros medios o revistas, siempre que el autor indique con claridad y en la primera nota a pie de página que el trabajo se publicó por primera vez en el Anuario, con indicación del número, año, páginas y DOI (si procede). La revista se vende impresa Bajo Demanda. 